TAMKANG JOURNAL OF MATHEMATICS

Volume 33, Number 2, Summer 2002

\title{
ON ABSOLUTE GENERALIZED NÖRLUND SUMMABILITY OF ORTHOGONAL SERIES
}

\author{
YASUO OKUYAMA
}

\begin{abstract}
In this paper, we shall prove a general theorem which contains two theorems on the absolute Nörlund summability and the absolute Riesz summability of orthogonal series.
\end{abstract}

1. Let $\sum_{n=0}^{\infty} a_{n}$ be a given infinite series with sequence of partial sums $\left\{s_{n}\right\}$. Let $p$ denote the sequence $\left\{p_{n}\right\}$. Given two sequences $p$ and $q$, the convolution $(p * q)$ is defined by

$$
(p * q)_{n}=\sum_{k=0}^{n} p_{n-k} q_{k}=\sum_{k=0}^{n} p_{k} q_{n-k} .
$$

When $(p * q)_{n} \neq 0$ for all $n$, the generalized Nörlund transform of the sequence $\left\{s_{n}\right\}$ is the sequence $\left\{t_{n}^{p, q}\right\}$ obtained by putting

$$
t_{p}^{p, q}=\frac{1}{(p * q)_{n}} \sum_{k=0}^{n} p_{n-k} q_{k} s_{k}
$$

If $\lim _{n \rightarrow \infty} t_{n}^{p, q}$ exists and is equal to $s$, then the series $\sum_{n=0}^{\infty} a_{n}$ or the sequence $\left\{s_{n}\right\}$ is said to be summable $\left(N, p_{n}, q_{n}\right)$ to the value $s$ and we write

$$
\sum_{n=0}^{\infty} a_{n}=s\left(N, p_{n}, q_{n}\right) \quad \text { or } \quad s_{n} \rightarrow s\left(N, p_{n}, q_{n}\right)
$$

(see Borwein [1]).

If the series $\sum_{n=0}^{\infty}\left|t_{n}^{p, q}-t_{n-1}^{p, q}\right|$ converges, then the series $\sum_{n=0}^{\infty} a_{n}$ is said to be summable $\left|N, p_{n}, q_{n}\right|$ and we write $\sum_{n=0}^{\infty} a_{n} \in\left|N, p_{n}, q_{n}\right|$ (see Tanaka [5]).

The method $\left|N, p_{n}, q_{n}\right|$ reduces to the absolute Nörlund method $\left|N, p_{n}\right|$ if $q_{n}=1$ for all $n$ and to the absolute Riesz method $\left|\bar{N}, q_{n}\right|$ if $p_{n}=1$ for all $n$. We know that $\left|N, p_{n}\right|$ mean or $\left|\bar{N}, q_{n}\right|$ includes as special case the absolute Cesàro mean and the absolute harmonic mean or the logarithmic mean, respectively. Finally $A$ denotes a positive absolute constant not the same.

Received May 22, 2001.

2000 Mathematics Subject Classification. 42C15, 40F05, 40G05.

Key words and phrases. Orthogonal series, Nörlund summability. 
2. Let $\left\{\varphi_{n}(x)\right\}$ be an orthonormal system defined in the internal $(a, b)$. We suppose that $f(x)$ belongs to $L^{2}(a, b)$ and

$$
f(x) \sim \sum_{n=0}^{\infty} a_{n} \varphi_{n}(x)
$$

We also write

$$
R_{n}=(p * q)_{n}, R_{n}^{j}=\sum_{k=j}^{n} p_{n-k} q_{k} \quad \text { and } \quad R_{n}^{n+1}=0 .
$$

Then we have

$$
R_{n}^{0}=R_{n}
$$

Further we put

$$
P_{n}=(p * 1)_{n}=\sum_{k=0}^{n} p_{k} \quad \text { and } \quad Q_{n}=(1 * q)_{n}=\sum_{k=0}^{n} q_{k} .
$$

Now we shall prove a general theorem on the absolute generalized Nörlund summability of the orthogonal series.

Theorem 1. If the series

$$
\sum_{n=1}^{\infty}\left\{\sum_{j=1}^{n}\left(\frac{R_{n}^{j}}{R_{n}}-\frac{R_{n-1}^{j}}{R_{n-1}}\right)^{2}\left|a_{j}\right|^{2}\right\}^{1 / 2}
$$

converges, then the orthogonal series

$$
\sum_{n=0}^{\infty} a_{n} \varphi_{n}(x)
$$

is summable $\left|N, p_{n}, q_{n}\right|$ almost everywhere.

Proof. Let $t_{n}^{p, q}(x)$ be the $n$-the $\left(N, p_{n}, q_{n}\right)$ mean of the series $\sum_{n=0}^{\infty} a_{n} \varphi_{n}(x)$. Then we have

$$
\begin{aligned}
t_{n}^{p, q}(x) & =\frac{1}{R_{n}} \sum_{k=0}^{n} p_{n-k} q_{k} s_{k}(x) \\
& =\frac{1}{R_{n}} \sum_{k=0}^{n} p_{n-k} q_{k} \sum_{j=0}^{k} a_{j} \varphi_{j}(x) \\
& =\frac{1}{R_{n}} \sum_{j=0}^{n} a_{j} \varphi_{j}(x) \sum_{k=j}^{n} p_{n-k} q_{k} \\
& =\frac{1}{R_{n}} \sum_{j=0}^{n} R_{n}^{j} a_{j} \varphi_{j}(x),
\end{aligned}
$$


where $s_{n}(x)=\sum_{k=0}^{n} a_{k} \varphi_{k}(x)$.

Thus we obtain

$$
\begin{aligned}
t_{n}^{p, q}(x)-t_{n-1}^{p, q}(x) & =\frac{1}{R_{n}} \sum_{j=0}^{n} R_{n}^{j} a_{j} \varphi_{j}(x)-\frac{1}{R_{n-1}} \sum_{j=0}^{n-1} R_{n-1}^{j} a_{j} \varphi_{j}(x) \\
& =\frac{1}{R_{n}} \sum_{j=1}^{n} R_{n}^{j} a_{j} \varphi_{j}(x)-\frac{1}{R_{n-1}} \sum_{j=1}^{n-1} R_{n-1}^{j} a_{j} \varphi_{j}(x) \\
& =\frac{1}{R_{n}} \sum_{j=1}^{n} R_{n}^{j} a_{j} \varphi_{j}(x)-\frac{1}{R_{n-1}} \sum_{j=1}^{n} R_{n-1}^{j} a_{j} \varphi_{j}(x) \\
& =\sum_{j=1}^{n}\left(\frac{R_{n}^{j}}{R_{n}}-\frac{R_{n-1}^{j}}{R_{n-1}}\right) a_{j} \varphi_{j}(x) .
\end{aligned}
$$

Using the Schwarz's inequality and the orthogonality, we obtain

$$
\begin{aligned}
\int_{a}^{b}\left|\Delta t_{n}^{p, q}(x)\right| d x & \leq(b-a)^{1 / 2}\left\{\int_{a}^{b}\left|\Delta t_{n}^{p, q}(x)\right|^{2} d x\right\}^{1 / 2} \\
& =(b-a)^{1 / 2}\left\{\sum_{j=1}^{n}\left(\frac{R_{n}^{j}}{R_{n}}-\frac{R_{n-1}^{j}}{R_{n-1}}\right)^{2}\left|a_{j}\right|^{2}\right\}^{1 / 2}
\end{aligned}
$$

and then

$$
\sum_{n=1}^{\infty} \int_{a}^{b}\left|\Delta t_{n}^{p, q}(x)\right| d x \leq(b-a)^{1 / 2} \sum_{n=1}^{\infty}\left\{\sum_{j=1}^{n}\left(\frac{R_{n}^{j}}{R_{n}}-\frac{R_{n-1}^{j}}{R_{n-1}}\right)^{2}\left|a_{j}\right|^{2}\right\}^{1 / 2}
$$

which is convergent by the assumption and from the Beppo-Leni Lemma we complete the proof.

We can obtain two the following corollaries from our theorem.

Corollary 1.([2,4]) If the series

$$
\sum_{n=1}^{\infty} \frac{p_{n}}{P_{n} P_{n-1}}\left\{\sum_{j=1}^{n} p_{n-j}^{2}\left(\frac{P_{n}}{p_{n}}-\frac{P_{n-j}}{p_{n-j}}\right)^{2}\left|a_{j}\right|^{2}\right\}^{1 / 2}
$$

converges, then the orthogonal series

$$
\sum_{n=0}^{\infty} a_{n} \varphi_{n}(x)
$$

is summable $\left|N, p_{n}\right|$ almost everywhere.

Proof. The proof follows from our theorem and the fact that

$$
\frac{R_{n}^{j}}{R_{n}}-\frac{R_{n-1}^{j}}{R_{n-1}}=\frac{P_{n-j}}{P_{n}}-\frac{P_{n-1-j}}{P_{n-1}}
$$




$$
\begin{aligned}
& =\frac{1}{P_{n} P_{n-1}}\left(P_{n-1} P_{n-j}-P_{n} P_{n-1-j}\right) \\
& =\frac{1}{P_{n} P_{n-1}}\left\{\left(P_{n}-p_{n}\right) P_{n-j}-P_{n}\left(P_{n-j}-p_{n-j}\right)\right\} \\
& =\frac{1}{P_{n} P_{n-1}}\left(P_{n} P_{n-j}-p_{n} P_{n-j}-P_{n} P_{n-j}+p_{n-j} P_{n}\right) \\
& =\frac{p_{n}}{P_{n} P_{n-1}}\left(\frac{P_{n}}{p_{n}}-\frac{P_{n-j}}{p_{n-j}}\right) p_{n-j} \quad \text { for all } q_{n}=1 .
\end{aligned}
$$

Corollary 2.([3]) If the series

$$
\sum_{n=1}^{\infty} \frac{q_{n}}{Q_{n} Q_{n-1}}\left\{\sum_{j=1}^{n} Q_{j-1}^{2} a_{j}^{2}\right\}^{1 / 2}
$$

convergeces, then the orthogonal series

$$
\sum_{n=0}^{\infty} a_{n} \varphi_{n}(x)
$$

is summable $\left|\bar{N}, q_{n}\right|$ almost everywhere.

Proof. The proof follows from theorem 1 and the fact that

$$
\begin{aligned}
\frac{R_{n}^{j}}{R_{n}}-\frac{R_{n-1}^{j}}{R_{n-1}} & =\frac{Q_{n}-Q_{j-1}}{Q_{n}}-\frac{Q_{n-1}-Q_{j-1}}{Q_{n-1}} \\
& =Q_{j-1}\left(\frac{1}{Q_{n}}-\frac{1}{Q_{n-1}}\right) \\
& =-\frac{q_{n} Q_{j-1}}{Q_{n} Q_{n-1}} \quad \text { for all } p_{n}=1 .
\end{aligned}
$$

For the application of these corollaries, see Okuyama [2,3,4].

Furthermore, if we put

$$
w(j)=\frac{1}{j} \sum_{n=j}^{\infty} n^{2}\left(\frac{R_{n}^{j}}{R_{n}}-\frac{R_{n-1}^{j}}{R_{n-1}}\right)^{2},
$$

then we have the following theorem from theorem 1.

Theorem 2. Let $\{\Omega(n)\}$ be a positive sequence such that $\{\Omega(n) / n\}$ is a nonincreasing sequence and the series $\sum_{n=1}^{\infty} \frac{1}{n \Omega(n)}$ converges. Let $\left\{p_{n}\right\}$ and $\left\{q_{n}\right\}$ be nonnegative. If the series $\sum_{n=1}^{\infty}\left|a_{n}\right|^{2} \Omega(n) w(n)$ converges, then the orthogonal series $\sum_{n=0}^{\infty}$ $a_{n} \varphi_{n}(x)$ is summable $\left|N, p_{n}, q_{n}\right|$ almost everywhere, where $w(n)$ is defined by (2). 
Proof. We have by Schwarz inequality

$$
\begin{aligned}
\sum_{n=1}^{\infty} \int_{a}^{b}\left|\Delta t_{n}^{p, q}(x)\right| d x & \leq A \sum_{n=1}^{\infty}\left\{\sum_{j=1}^{n}\left(\frac{R_{n}^{j}}{R_{n}}-\frac{R_{n-1}^{j}}{R_{n-1}}\right)^{2}\left|a_{j}\right|^{2}\right\}^{1 / 2} \\
& =A \sum_{n=1}^{\infty} \frac{1}{n^{1 / 2} \Omega(n)^{1 / 2}}\left\{n \Omega(n) \sum_{j=1}^{n}\left(\frac{R_{n}^{j}}{R_{n}}-\frac{R_{n-1}^{j}}{R_{n-1}}\right)^{2}\left|a_{j}\right|^{2}\right\}^{1 / 2} \\
& \leq A\left\{\sum_{n=1}^{\infty} \frac{1}{n \Omega(n)}\right\}^{1 / 2}\left\{\sum_{n=1}^{\infty} n \Omega(n) \sum_{j=1}^{n}\left(\frac{R_{n}^{j}}{R_{n}}-\frac{R_{n-1}^{j}}{R_{n-1}}\right)^{2}\left|a_{j}\right|^{2}\right\}^{1 / 2} \\
& \leq A\left\{\sum_{j=1}^{\infty}\left|a_{j}\right|^{2} \sum_{n=j}^{\infty} n \Omega(n)\left(\frac{R_{n}^{j}}{R_{n}}-\frac{R_{n-1}^{j}}{R_{n-1}}\right)^{2}\right\}^{1 / 2} \\
& \leq A\left\{\sum_{j=1}^{\infty}\left|a_{j}\right|^{2} \frac{\Omega(j)}{j} \sum_{n=j}^{\infty} n^{2}\left(\frac{R_{n}^{j}}{R_{n}}-\frac{R_{n-1}^{j}}{R_{n-1}}\right)^{2}\right\}^{1 / 2} \\
& =A\left\{\sum_{j=1}^{\infty}\left|a_{j}\right|^{2} \Omega(j) w(j)\right\}^{1 / 2}<\infty
\end{aligned}
$$

by virtue of the hypotheses of Theorem 2 . Thus this completes the proof of Theorem 2 from the same reason of the proof of Theorem 1.

\section{References}

[1] D. Borwein, On product of sequences, J. London Math. Soc. 33(1958), 352-357.

[2] Y. Okuyama, On the absolute Nörlund summability of orthogonal series, Proc. Japan Acad. 54(1978), 113-118.

[3] Y. Okuyama and T. Tsuchikura, On the absolute Riesz summability of orthogonal series, Analysis Math, 7(1981), 199-208.

[4] Y. Okuyama, Absolute summability of Fourier series and orthogonal series, Lecture Notes in Math. No.1067(1984), Springer-Verlag.

[5] M. Tanaka, On generalized Nörlund methods of summability, Bull. Austral. Math. Soc. $\mathbf{1 9}(1978), 381-402$.

Department of Mathematics, Faculty of Engineering, Shinshu University, 4-17-1 Wakasato, Nagano 380-8553, Japan. 